\title{
A rare case of primary fallopian tube carcinoma in a woman with post-menopausal bleeding
}

\author{
P. G. Paul*, Anjana Annal, K. Anusha Chowdary, Manali Shilotri, \\ George Paul, Madhumathi Degapudi
}

Paul's Hospital, Centre for Advanced Gynecological Endoscopy and Infertility, Kochi, Kerala, India

Received: 09 July 2021

Revised: 05 August 2021

Accepted: 06 August 2021

\section{*Correspondence:}

Dr. P. G. Paul,

E-mail: drpaulpg@gmail.com

Copyright: () the author(s), publisher and licensee Medip Academy. This is an open-access article distributed under the terms of the Creative Commons Attribution Non-Commercial License, which permits unrestricted non-commercial use, distribution, and reproduction in any medium, provided the original work is properly cited.

\begin{abstract}
Primary fallopian tube carcinoma (PFTC) is a rare tumor accounting for about $0.5 \%$ of all gynecological cancers. The rarity of the tumor with its non-specific clinical presentation and radiological findings lead to a low suspicion of PFTC by the attending clinician. We present a case of this tumor in a 62 years post-menopausal parous woman who presented with intermittent vaginal bleeding with normal clinical examination and an adnexal mass on USG and MRI. She underwent laparosopic total hysterectomy with bilateral salpingo-oophorectomy, infracolic omentectomy and staging biopsies followed by adjuvant chemotherapy. Primary fallopian tube carcinoma should be considered in the differential diagnosis of peri and postmenopausal women who present with an adnexal mass, unexplained bleeding per vaginum, pelvic pain, or a cervical smear with abnormal glandular cells. The treatment is similar to epithelial ovarian carcinoma, which includes total hysterectomy with bilateral salpingo-oophorectomy and comprehensive surgical staging.
\end{abstract}

Keywords: Fallopian tube carcinoma, Post-menopausal bleeding, Adnexal mass

\section{INTRODUCTION}

Primary fallopian tube carcinoma (PFTC) is a rare tumor accounting for about $0.5 \%$ of all gynecological cancers. ${ }^{1}$ However, its incidence may be higher than estimated as molecular, histologic, and genetic evidence shows that almost $80 \%$ of tumors that were previously classified as high-grade serous carcinomas of the ovary or peritoneum may have an origin in the fimbriae of the fallopian tubes. ${ }^{2}$ As a result, the epithelial ovarian carcinomas, fallopian tube and peritoneal cancers are considered collectively and have the same staging and similar management protocols. The rarity of this tumor with its non-specific clinical presentation and radiological findings lead to a low suspicion of PFTC by the attending clinician. Thus, PFTC is usually diagnosed incidentally, intra-operatively or on histopathology. The overall survival of patients with PFTC is generally low. We present a case of this rare tumor in a 62-years-old postmenopausal parous woman who presented with vaginal bleeding and a complex adnexal mass.

\section{CASE REPORT}

A 62-years-old para 1, living 1 postmenopausal woman presented to us with intermittent vaginal bleeding without any other symptoms. General, abdominal, and pelvic examination was normal. The pelvic ultrasonography showed a left adnexal complex cyst of $5.7 \times 3.4 \mathrm{~cm}$ with solid components of $3.2 \times 2 \mathrm{~cm}$ and $2.3 \times 1.7 \mathrm{~cm}$ and high vascularity with a resistance index of 0.5 (Figure 1$)$. The uterus, endometrial thickness $(3 \mathrm{~mm})$, right adnexa were normal and there was no evidence of ascites. Tumor markers were normal; CA-125: $9.1 \mathrm{U} / \mathrm{ml}$ and Inhibin A: $<1 \mathrm{pg} / \mathrm{ml}$. The ovarian malignancy risk stratification by IOTA-ADNEX model suggested a $67.5 \%$ chance of a 
benign tumor. MRI suggested a normal-sized uterus with subcentimetric myomas, normal right ovary, and a lobulated solid lesion of $3.5 \times 2 \mathrm{~cm}$ in the left adnexa, with a differential diagnosis of a degenerating fibroid or an ovarian mass. Pap smear was normal and endometrial biopsy suggested an atrophic endometrium. She underwent a laparoscopy which showed a left complex tubo-ovarian mass of $4 \mathrm{~cm}$ in the distal half of the fallopian tube (Figure 2). The uterus and right adnexa were normal. There was no evidence of any implants, deposits or free fluid in the pelvis or abdomen. The omentum was normal and there was no lymphadenopathy. Considering a $32.5 \%$ chance of malignancy by the IOTA-ADNEX model, we performed a total laparoscopic hysterectomy with bilateral salpingo-oophorectomy, infracolic omentectomy, staging biopsies and fluid cytology. Post-operative recovery was uneventful. Histopathology was suggestive of high grade papillary serous PFTC of the left tube invading into the muscularis layer, no serosal or left ovarian involvement, and other sites free from the tumor (Figure 3). There was compact papillary, nesting and glandular growth pattern in the tumor with the cells displaying pleiomorphic nuclei having abnormal mitotic activity. Considering her FIGO stage 1c1 (due to surgical spillage), she was given 6 cycles of adjuvant chemotherapy with cisplatin and paclitaxel. At her last follow-up at 1-year post-surgery, clinical examination, ultrasonography, and PET scan were normal and she was doing well.

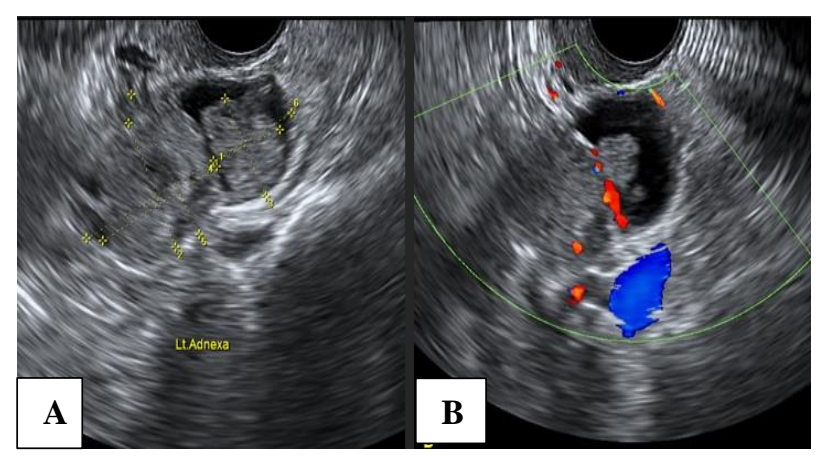

Figure 1: Ultrasonography showing left adnexal mass (A) with a solid area; (B) with color flow.

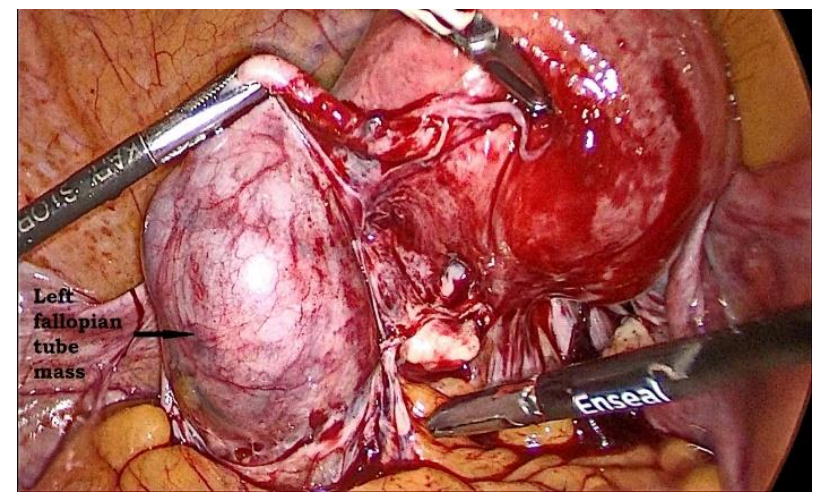

Figure 2: Laparoscopy showing left tubal mass.

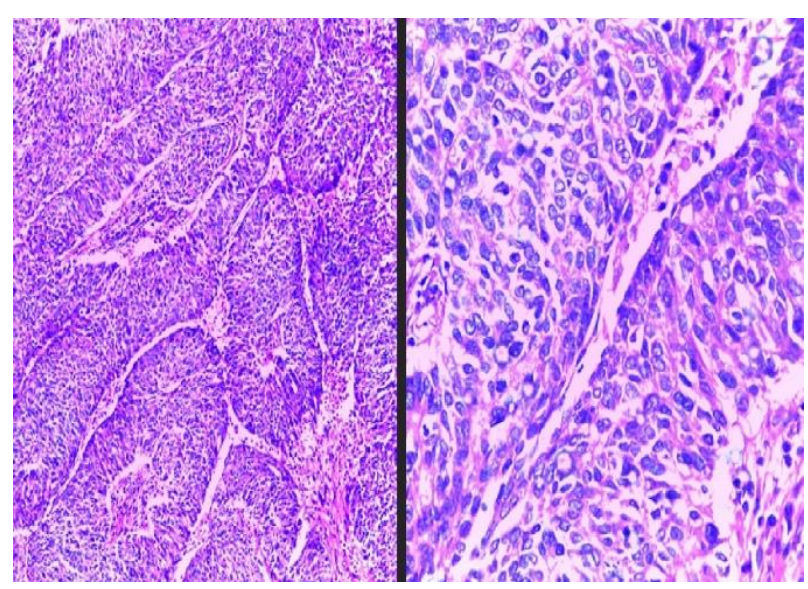

Figure 3: Histopathology of primary high-grade serous carcinoma of the fallopian tube showing papillary, nesting and glandular pattern with pleomorphic nuclei and abnormal mitotic activity.

\section{DISCUSSION}

PFTCs are rare tumors of the female genital tract with incidence being 0.36 to 0.41 per 100,000 women per year. ${ }^{3}$ With the recent change in our understanding of the origin of serous carcinomas, several cancers of the ovaries may actually have been fallopian tube carcinomas, thus causing under-estimation of their incidence.

The presenting symptoms are non-specific such as abdominal pain, vaginal discharge, abnormal uterine bleeding, and lump in abdomen. The pathognomonic Latzko's triad of intermittent vaginal discharge, colicky pain relieved after vaginal discharge and abdominal or pelvic mass is noted in less than $15 \%$ of cases. ${ }^{4}$ It is postulated that PFTC may be diagnosed in early stages because of abdominal pain caused by tubal distension and a shorter course of symptoms as compared to ovarian carcinomas. $^{5}$

The mean age of presentation is about 57 years (ranging from 42 to 72 years). ${ }^{1}$ Risk-factors are similar to epithelial ovarian carcinomas such as infertility, low parity and germ-line BRCA-1 and BRCA-2 mutations while use of oral contraceptive pills provides a significantly protective effect. $^{6}$ On ultrasound, the typical features of PFTC are said to be a sausage-shaped cystic structure with solid tissue protruding into it like a papillary projection, a sausage-shaped cystic structure with a large solid component filling part of the cyst cavity and an ovoid or oblong completely solid mass. ${ }^{7}$

Pre-operative serum CA-125 levels may be raised $(>35$ $\mathrm{mIU} / \mathrm{ml}$ ) and are seen in $85.3 \%$ of cases. ${ }^{8}$ However, in our case, CA-125 levels were normal, thus a high index of suspicion is required. Persistent abnormal glandular cells on cervical smears with normal endometrial and endocervical biopsies should raise the suspicion of PFTC. Cervical smears have been suggested as valuable tools for screening for PFTC. ${ }^{9}$ The diagnostic criteria for PFTC are: 
grossly, the main tumor is in the tube and arises from the endosalpinx; the histological pattern reproduces the epithelium of tubal mucosa; transition from benign to malignant tubal epithelium should be demonstrated, and ovaries and endometrium are either normal or have a much smaller tumor volume than that of the tube..$^{10,11}$

Fallopian tube, ovarian and peritoneal carcinomas have such similar patterns of spread, clinical presentation, surgical responsiveness and chemosensitivity that the same treatment protocol is applied to all three diseases viz. total hysterectomy with bilateral salpingo-oophorectomy and comprehensive surgical staging with chemoradiation depending on the stage. Factors influencing survival are the patient age, FIGO stage of disease and residual tumor after initial surgery. Although similar to epithelial ovarian carcinoma, in PFTC there are microscopic metastases to distant sites even in early stages and it often tends to recur in retroperitoneal nodes and distant sites. Thus, adjuvant chemotherapy is recommended in early-stage disease. ${ }^{8}$

\section{CONCLUSION}

Primary fallopian tube carcinoma should be considered in the differential diagnosis of peri and postmenopausal women who present with an adnexal mass, unexplained bleeding per vaginum, pelvic pain, or a cervical smear with abnormal glandular cells. The treatment is similar to epithelial ovarian carcinoma, which includes total hysterectomy with bilateral salpingo-oophorectomy and comprehensive surgical staging with adjuvant chemotherapy, depending on the stage.

\section{ACKNOWLEDGMENTS}

We would like to thank Dr. Iona Leekha Mathew, Senior Consultant (Pathology) at VPS Lakeshore Hospital, Kochi, India for her aid in the histopathological findings.

Funding: No funding sources

Conflict of interest: None declared

Ethical approval: Not required

\section{REFERENCES}

1. Ma Y, Duan W. Clinical and survival analysis of 36 cases of primary fallopian tube carcinoma. World $\mathrm{J}$ Surg Oncol. 2014;12:311.

2. Berek JS, Kehoe ST, Kumar L, Friedlander M. Cancer of the ovary, fallopian tube, and peritoneum. Int $\mathrm{J}$ Gynaecol Obstet. 2018;143(2):59-78.

3. Stasenko M, Fillipova O, Tew WP. Fallopian Tube Carcinoma. J Oncol Pract. 2019;15(7):375-82.

4. Vyas MN, Rai S, Manjeera L, Shetty D. Bilateral primary fallopian tube carcinoma with the classical clinical features: a case report. J Clin Diagn Res. 2013;7(4):726-8.

5. Moore KN, Moxley KM, Fader AN, Axtell AE, Rocconi RP, Abaid LN, et al. Serous fallopian tube carcinoma: a retrospective, multi-institutional casecontrol comparison to serous adenocarcinoma of the ovary. Gynecol Oncol. 2007;107(3):398-403.

6. Rexhepi M, Trajkovska E, Ismaili H, Besimi F, Rufati N. Primary Fallopian Tube Carcinoma: A Case Report and Literature Review. Open Access Maced J Med Sci. 2017;5(3):344-8.

7. Rexhepi M, Trajkovska E, Ismaili H, Besimi F, Rufati N. Primary Fallopian Tube Carcinoma: A Case Report and Literature Review. Open Access Maced J Med Sci. 2017;5(3):344-8.

8. Gadducci A, Landoni F, Sartori E, Maggino T, Zola $\mathrm{P}$, Gabriele A, et al. Analysis of treatment failures and survival of patients with fallopian tube carcinoma: a cooperation task force (CTF) study. Gynecol Oncol. 2001;81(2):150-9.

9. Ural UM, Balik G, Tekin YB, Sehitoglu I, Bedir R, Sahin FK. Primary fallopian tube carcinoma diagnosed preoperatively by cervical smear. Ann Saudi Med. 2014;34(5):444-6.

10. Sedlis A. Carcinoma of the fallopian tube. Surg Clin North Am. 1978;58:121-9.

11. Hu CY, Taymor ML, Hertig AT. Primary carcinoma of the fallopian tube. Am J Obstet Gynecol. 1950;50:58-67.

Cite this article as: Paul $\mathrm{PG}$, Annal A, Chowdary KA, Shilotri M, Paul G, Degapudi M. A rare case of primary fallopian tube carcinoma in a woman with post-menopausal bleeding. Int J Reprod Contracept Obstet Gynecol 2021;10:3630-2. 